\title{
Adult Ixodes scapularis Ticks in a Lyme Disease Hot Spot are Vectors for Emerging Pathogens
}

\author{
Vida R. Irani* and Jeffery L. Larkin \\ Indiana University of Pennsylvania, Department of Biology, 975 Oakland Ave, Indiana, PA 15705
}

\begin{abstract}
Received: September 9, 2016; Accepted: October 21, 2016; Published: October 24, 2016
*Corresponding author: Vida R. Irani, Indiana University of Pennsylvania, Department of Biology, 975 Oakland Ave, Indiana, PA 15705, Tel no: 724-357-2625; E-mail: virani@iup.edu
\end{abstract}

\begin{abstract}
A 2015 Center for Disease Control and Prevention study concluded that for northeastern United States, Lyme, a debilitating tick-borne disease is expanding west ward with counties in western Pennsylvania identified as high-incidence areas. Lyme's primary vector, the black-legged tick (Ixodes scapularis), is also the host vector to at least four additional human pathogens. Thus, we tested adult $I$. scapularis ticks for co-infection in nine recreational areas located in a western Pennsylvania county categorized as high-incidence for Lyme. Specifically, we tested ticks for the Lyme disease pathogen, Borrelia burgdorferi, and two other emerging blood-borne pathogens, Babesia microti and Anaplasma phagocytophilum. Our analyses suggest that the three pathogens were independent in their distribution with $B$. burgdorferi present in 30\%, B. microti in $27 \%$ and A. phagocytophilum in $4 \%$ of the tested I. scapularis samples. Forty-two percent ( $\mathrm{n}=106$ / 252) of all $I$. scapularis ticks tested carried only one pathogen, of which only half $(51 \%)$ of those carried B. burgdorferi and the rest carried either B. microti or A. phagocytophilum. Fifty-one percent of $I$. scapularis ticks tested carried at least one of the above three pathogens, with $9 \%$ of the ticks carrying more than one pathogen, of which $6 \%$ were co-infected with B. burgdorferi and B. microti. In recreational areas we sampled, a single bite from $I$. scapularis has the potential to transmit multiple pathogens which could result in concurrent infections and contribute to a variety of clinical symptoms not typically seen in Lyme disease patients. Our results considered in combination with previously published information on underreporting of human Lyme disease warrants the need for public health officials to be vigilant in the surveillance for tick-borne coinfections especially in areas endemic for Lyme.
\end{abstract}

\section{Introduction}

Vector-borne diseases are a major cause of human morbidity and mortality [1,2]. The black-legged tick, Ixodes scapularis (formerly known in the northeastern United States as the deer tick, Ixodes dammini) is the host vector to at least five human pathogens-Anaplasma phagocytophilum, Babesia microti, Borrelia burgdorferi, Ehrlichia species and Powassan virus [3], acquired by the tick after a blood meal from a co-infected wildlife host/ reservoir or as a result of sequentially feeding on diverse infected wildlife reservoirs. In addition to sharing the I. scapularis tick as a common vector, these pathogens can also share a common animal reservoir host, the white-footed mouse
(Peromyscus leucopus) [4-6]. Although Lyme disease is the leading vector-borne zoonotic disease caused by $B$. burgdorferi in the U.S., human co-infection with B. microti (Babesiosis) and $A$. phagocytophilum (Anaplasmosis) can occur in patients infected with B. burgdorferi [7-9]. Also, humans co-infected with more than one of the above mentioned zoonotic pathogens residing in an I. scapularis tick may be at risk of a harsher, more debilitating form of Lyme, Babesiosis or Anaplasmosis [7,10].

In recent years, Pennsylvania has led the United States in number of Lyme disease cases [1,11]. Because of human population density and proximity to the heavily populated areas of the northeastern U.S., there is a continuing misconception that Lyme disease cases are on the rise only in northeast Pennsylvania [12]. But over the past decade, many counties in western Pennsylvania have experienced an increase in incidence of Lyme Borreliosis in human adults and children [11] which is corroborated by the latest CDC finding that "The center of the high-incidence focus in the northeastern United States generally moved westward and northward, away from the coast of northern New Jersey and into east-central Pennsylvania" [13].

A combination of varying land use practices, diverse and abundant wildlife reservoirs (white-footed mouse, white-tailed deer, ground birds, eastern chipmunks, shrews), and recreational activities such as hiking, camping, fishing and hunting have increased the potential exposure of humans to I. scapularis ticks in western Pennsylvania. It is estimated that Pennsylvania's public recreational areas attract over 34 million visitors annually [14] and Pennsylvania ranks sixth in yearly sales of fishing licenses and second in yearly sales of hunting licenses [15]. Thus, the Commonwealth of Pennsylvania hosts a large human demographic that is at risk for exposure to multiple tick-borne pathogens.

The purpose of this study was to determine (i) the infection rates for A. phagocytophilum, B. microti and B. burgdorferi in I. scapularis collected in recreational areas of a western Pennsylvania county known to have a high-incidence for human Lyme disease; and (ii) the frequency of co-infections in these ticks which could translate to concurrent infections in humans via a single tick bite. Our results have added to the knowledge of 
the microbial community residing in I. scapularis ticks in hot spot areas, which is important for increasing human vigilance and for public health professionals to make more informed prevention, diagnoses, and treatment decisions.

\section{Materials and Methods}

\section{Tick collection}

Our nine study areas for questing I. scapularis adult tick collection (April-November 2013) are public conservation parks and lands in southwestern county of Indiana, Pennsylvania (Figure 1 inset) that are similarly forested and abundant with many mast producing trees such as oaks that provide abundant food resources for many mammalian vectors. Additionally, our study areas and the surrounding landscape contained an intermix of other land use types such as scattered shrub lands, natural gas wells, small managed grass openings, and park infrastructure such as roads and utility corridors which could result in the creation of edge habitats that contribute to I. scapularis tick survival and abundance by supporting the habitat preferred by many animal host reservoir species.

All ticks were collected by dragging a white cloth attached to a rope through vegetation and leaf litter along hiking trails and in adjacent forests. All ticks collected were immediately transferred to $70 \%$ ethanol and stored in a laboratory at the Indiana University of Pennsylvania (IUP, Indiana, Pennsylvania) until experimentation. Prior to pathogen testing, adult $I$ scapularis ticks were identified as either male or female based on morphological differences [16].

\section{DNA extraction and Polymerase Chain Reaction Analyses}

Due to susceptibility of all $I$. scapularis genomic DNA (gDNA) to quick degradation (unpublished results), we modified our DNA extraction protocols to isolate gDNA from I. scapularis ticks. The Dilution and Storage protocol using dilution buffer and DNA release additive of the Thermo Scientific Phire Tissue Direct PCR master mix kit (Thermo Scientific, Waltham, MA) extracted gDNA for PCR directly from the tick without proteinase K digestion, and purification steps (Thermo Fisher Scientific, Inc). Each tick was placed into $20.5 \mu \mathrm{l}$ of dilution buffer/ DNA release additive mix. The reaction was incubated at $25^{\circ} \mathrm{C}$ for $5 \mathrm{~min}$ and then placed in a $98^{\circ} \mathrm{C}$ water bath for $2 \mathrm{~min}$. The tubes were spun in a centrifuge and $1 \mu \mathrm{l}$ of the supernatant was used to measure DNA concentration using the NanoDrop (Thermo Fisher Scientific, Inc). All DNA samples were then stored at $-20^{\circ} \mathrm{C}$ till further analyses. Following DNA extraction, we performed DNA amplification using conventional PCR technique with the T100 thermal cycler (Bio-Rad, Hercules, CA) on adult I. scapularis tick gDNA sample. Forward and Reverse primers specific for the I. scapularis tick and each of the three pathogens were custom made from Integrated DNA Technologies, Inc. (Coralville, IA). Positive controls were provided by past collaborators (see Acknowledgments), and two sets of negative controls (distilled water and a solution of dilution

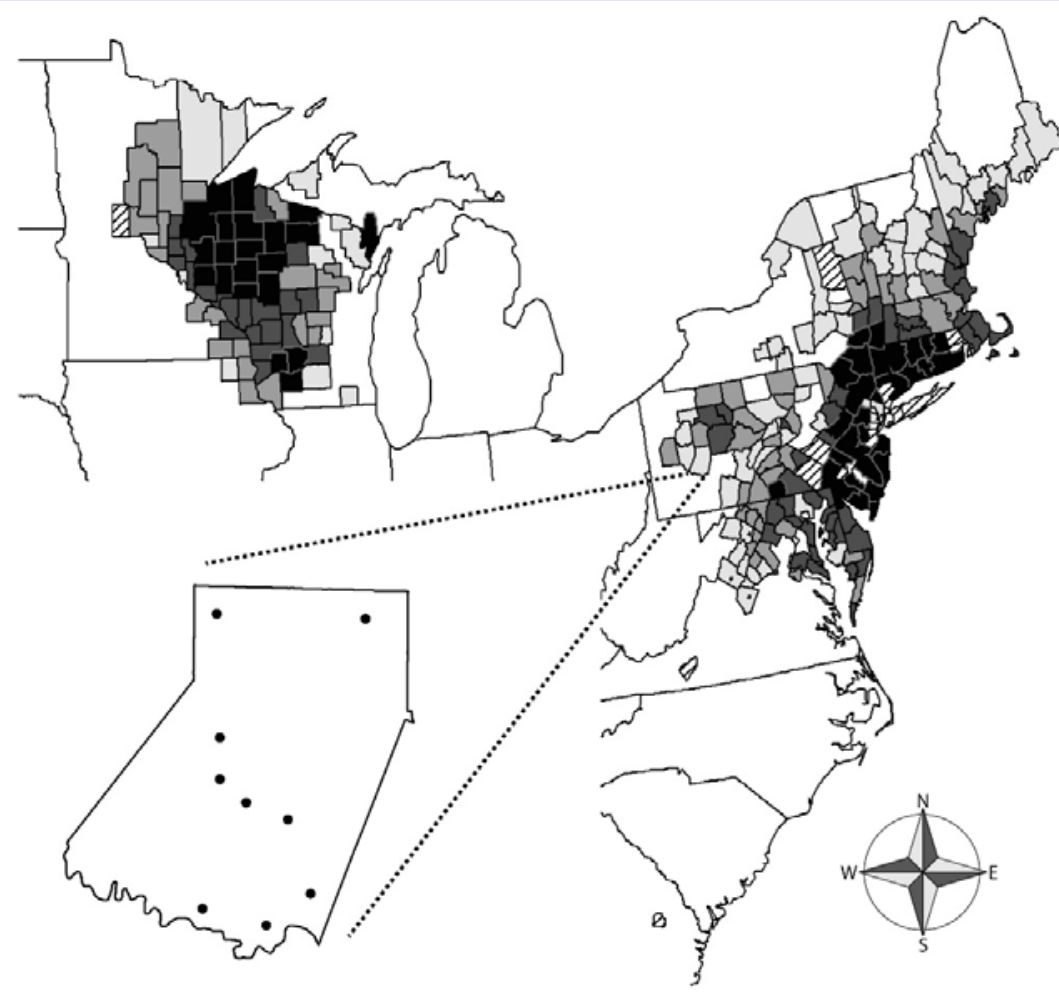

Period when county first achieved high-incidence status

1993-1997 1998-2002 2003-2007 2008-2012

Counties with high-incidence status $\geq 1$ period, but removed for subsequent periods $\mathbb{Z}$

Figure 1: Adapted with permission from Kugeler, et al. [13] of United States counties with high incidence of Lyme disease with inset of southwestern Pennsylvania county, location of the nine high risk recreational areas (closed circles), our Ixodes scapularis sampling sites. 
buffer/ DNA release additive mix) were used in place of extracted DNA to control for contamination.

To detect $A$. phagocytophilum, we used the ge3a-F/ ge10r-R primer set (GenBank accession number KJ942183.1). The forward primer was 5' CAC ATG CAA GTC GAA CGG ATT C 3' and the reverse primer were 5' TTC CGT TAA GAA GGA TCT AAT CTC C 3' amplifying a 932-base pair (bp) product specific for the $A$. phagocytophilum 16S rRNA gene. Amplification was performed as follows: $3 \mathrm{~min}$ at $98^{\circ} \mathrm{C}$ (one cycle) followed by 40 cycles for 5 sec at $98^{\circ} \mathrm{C}, 5 \mathrm{sec}$ at $55^{\circ} \mathrm{C}, 90 \mathrm{sec}$ at $72^{\circ} \mathrm{C}$. A. phagocytophilum was not further differentiated between the human pathogenic (AP$\mathrm{Ha}$ ) and the nonpathogenic (AP-variant 1) strains [12].

We used the PIRO-AF/ PIRO-BR primer set to detect $B$. microti [17]. The forward primer was 5' ATT ACC CAA TCC TGA CAC AGG G 3' and the reverse primer was 5' TTA AAT ACG AAT GCC CCC ACC 3' amplifying a 437-bp product specific for the $B$. microti 18S rRNA gene. Amplification was performed as follows: $3 \mathrm{~min}$ at $98^{\circ} \mathrm{C}$ (one cycle) followed by 40 cycles for $5 \mathrm{sec}$ at $98^{\circ} \mathrm{C}$, $5 \mathrm{sec}$ at $55^{\circ} \mathrm{C}, 30 \mathrm{sec}$ at $72^{\circ} \mathrm{C}$.

To detect B. burgdorferi, we used the FL6-F/ FL7-R primer set (GenBank accession number X63413.1). The forward primer was 5' TTC AGG GTC TCA AGC GTC TTG GAC T 3' and the reverse primer were 5' GCA TTT TCA ATT TTA GCA AGT GAT G 3' amplifying a 276-bp product specific for the B. burgdorferi flagellin gene [18]. Amplification was performed as follows: 3 $\min$ at $98^{\circ} \mathrm{C}$ (one cycle) followed by 40 cycles for $5 \mathrm{sec}$ at $98^{\circ} \mathrm{C}, 5$ sec at $55^{\circ} \mathrm{C}, 30 \mathrm{sec}$ at $72^{\circ} \mathrm{C}$.

To confirm that all PCR reactions had I. scapularis tick DNA, we amplified the $16 \mathrm{~S}$ rRNA gene of I. scapularis tick using the Iscap16SF/Iscap16SR primer set (Genbank accession number KF146646). The forward primer was 5' CGG TCT GAA CTC AGA TCA AG 3' and the reverse primer was 5' GGG ACA AGA AGA CCC TAT G 3' amplifying a 320-bp product specific for the $16 \mathrm{~S}$ rRNA gene of the I. scapularis tick.

All PCR products were separated by horizontal gel electrophoresis on a 1.2\% Tris-acetate EDTA (TAE) ethidium bromide agarose gel followed by analyses using a Gel Doc XR
Imager (Bio-Rad, Hercules, CA).

\section{Statistical analyses}

Data were tabulated and analyzed in Microsoft Excel (Microsoft Corp; Redmond, WA) and SPSS (IBM Corp.; Armonk, NY; Ver. 23). Data summarizations included assessment of pathogen frequencies within and across tick genders. Primary analyses were conducted using Chi-square tests to assess tick gender differences in pathogen frequencies. Chi-square tests also were used to test for the non-independence of multiple pathogens within ticks. Predicted frequencies of tick pathogen combinations were estimated by the combinations of their independent assortments $[18,19]$.

\section{Results}

Of the 252 adult $I$. scapularis ticks tested, fifty-one percent $(128 / 252)$ were infected with at least one of the three microbial species with B. burgdorferi being present in 30\% (76/ 252) of tick samples, followed by B. microti $(27 \%, 69 / 252)$ and $A$. phagocytophilum (4\%, 9/252). Forty two percent (105/ 252) ticks tested were infected with only one of the three pathogens with approximately half of those ticks (51\%, 54/ 105) infected with B. burgdorferi and the rest carried either B. microti $(47 \%, 49$ / 105 ) or A. phagocytophilum (2\%, 2/ 105). Nine percent (23/252) ticks tested were infected with at least two of the three pathogens of which 6\% (16/252) were co-infected with B. burgdorferi $+B$. microti, 1\% (3/ 252) with B. burgdorferi + A. phagocytophilum, $<1 \%(1 / 252)$ with B. microti + A. phagocytophilum. Forty-nine percent of ticks tested (124/252) were uninfected and 1\% (3/ $252)$ infected with all three pathogens. Male and female ticks were marginally different in their B. burgdorferi $\left(\chi^{2}=3.71, \mathrm{df}=\right.$ $1, p=0.054)$ and A. phagocytophilum $\left(\chi^{2}=3.11, \mathrm{df}=1, p=0.078\right)$, but not in their B. microti $\left(\chi^{2}=0.30, \mathrm{df}=1, p=0.584\right.$; Table 1$)$ prevalence rates. Males and females did not differ in the relative proportions of ticks carrying, 0,1 , or $>1$ pathogen $\left(\chi^{2}=2.36\right.$, $\mathrm{df}=2, p=0.307$ ) nor in the overall frequency of ticks carrying pathogens of any kind $\left(\chi^{2}=1.22, \mathrm{df}=1, p=0.269\right.$; Table 1$)$.

The three pathogens were relatively independent in their distribution among ticks, both in the actual combinations of

Table 1: Comparative prevalence rate of Borrelia burgdorferi, Babesia microti and Anaplasma phagocytophilum in adult male and female Ixodes scapularis collected from nine public recreational areas in southwestern Pennsylvania.

\begin{tabular}{|c|c|c|c|c|c|c|c|c|c|}
\hline \multirow[t]{2}{*}{ Pathogens in I. scapularis } & \multicolumn{2}{|c|}{ total } & \multicolumn{2}{|c|}{$\mathbf{M}$} & \multicolumn{2}{|c|}{$\mathbf{F}$} & \multicolumn{3}{|c|}{ M vs F } \\
\hline & $\mathbf{n}$ & rate & $\mathbf{n}$ & rate & $\mathbf{n}$ & rate & Chi square & df & $p$ \\
\hline B. burgdorferi & 76 & 0.302 & 23 & 0.232 & 53 & 0.346 & 3.71 & 1 & 0.054 \\
\hline B. microti & 69 & 0.274 & 29 & 0.293 & 40 & 0.261 & 0.30 & 1 & 0.584 \\
\hline A. phagocytophilum & 9 & 0.036 & 1 & 0.010 & 8 & 0.052 & 3.11 & 1 & 0.078 \\
\hline no pathogens & 124 & 0.492 & 53 & 0.535 & 71 & 0.464 & \multirow{3}{*}{2.36} & \multirow{3}{*}{2} & \multirow{3}{*}{0.307} \\
\hline only 1 pathogen & 105 & 0.417 & 40 & 0.404 & 65 & 0.425 & & & \\
\hline more than 1 pathogen & 23 & 0.091 & 6 & 0.061 & 17 & 0.111 & & & \\
\hline at least 1 pathogen & 128 & 0.508 & 46 & 0.465 & 82 & 0.536 & 1.22 & 1 & 0.269 \\
\hline
\end{tabular}


Table 2: Observed and expected prevalence rates of Borrelia burgdorferi, Babesia microti and Anaplasma phagocytophilum in adult Ixodes scapularis collected from nine public recreational areas in southwestern Pennsylvania.

\begin{tabular}{|c|c|c|c|c|c|c|c|}
\hline \multirow[t]{2}{*}{ Pathogens in I. scapularis } & \multicolumn{2}{|c|}{ Observed } & \multicolumn{2}{|c|}{ Expected } & \multicolumn{3}{|c|}{ Observed vs. expected } \\
\hline & (n) & rate & (n) & rate & Chi square & df & $p$ \\
\hline B. burgdorferi $(\mathrm{Bb})$ & 76 & 0.302 & -- & -- & \multirow[b]{2}{*}{--} & \multirow[b]{2}{*}{--} & \multirow[b]{2}{*}{--} \\
\hline B. microti $(\mathrm{Bm})$ & 69 & 0.274 & -- & -- & & & \\
\hline $\mathrm{Bb}+\mathrm{Bm}$ & 16 & 0.063 & 20.81 & 0.083 & \multirow{4}{*}{2.36} & \multirow{4}{*}{3} & \multirow{4}{*}{0.501} \\
\hline $\mathrm{Bb}+\mathrm{Ap}$ & 3 & 0.012 & 2.71 & 0.011 & & & \\
\hline $\mathrm{Bm}+\mathrm{Ap}$ & 1 & 0.004 & 2.46 & 0.010 & & & \\
\hline$B b+B m+A p$ & 3 & 0.012 & 0.74 & 0.003 & & & \\
\hline no pathogens & 124 & 0.492 & 123.24 & 0.489 & \multirow{3}{*}{0.33} & \multirow{3}{*}{2} & \multirow{3}{*}{0.850} \\
\hline only 1 pathogen & 105 & 0.417 & 102.02 & 0.405 & & & \\
\hline more than 1 pathogen & 23 & 0.091 & 26.73 & 0.106 & & & \\
\hline at least 1 pathogen & 128 & 0.508 & 128.76 & 0.511 & -- & -- & -- \\
\hline
\end{tabular}

pathogens present in the ticks sampled $\left(\chi^{2}=2.36, \mathrm{df}=3, p=0.501\right)$ as well as in the relative number of pathogens observed within tick samples ( $\chi^{2}=0.33, \mathrm{df}=2, p=0.850$; Table 2). B. burgdorferi was found unevenly among the nine recreational areas sampled $\left(\chi^{2}=48.19, \mathrm{df}=8, p<0.001\right)$ whereas B. microti was not different in prevalence among areas sampled $\left(\chi^{2}=5.89, \mathrm{df}=8, p=0.660\right)$.

\section{Discussion}

To our knowledge, this study is the most comprehensive report of prevalence of three microbial pathogens, B. burgdorferi, B. microti and A. phagocytophilum in I. scapularis ticks tested from recreational areas in a region recently designated as "a center of high incidence focus" for human Lyme disease [13, Figure 1]. The three most striking patterns observed from our study are (i) 51\% of the 252 total I. scapularis ticks tested were infected with at least one of the three microbial pathogens; (ii) $42 \%$ of the 252 total $I$. scapularis ticks tested were infected with only one pathogen of which only 51\% (105/ 252) were identified as carrying the Lyme disease pathogen B. burgdorferi, while the other $49 \%$ of the ticks were infected with non-Borreliosis pathogens, causative agents of emerging blood-borne debilitating diseases; and iii) $1 \%$ of the ticks sampled had the potential to transmit all three pathogens via a single bite to humans.

Nine percent of the ticks sampled were co-infected with more than one pathogen which is not surprising given that zoonotic pathogens share a common I. scapularis tick vector and small mammal reservoirs [4-6]. We saw no evidence that coinfection occurred more or less often than expected based upon the co-infections predicted by combinations of the pathogens independent frequencies. Our coinfection rates for B. burgdorferi
+ A. phagocytophilum and B. microti+ A. phagocytophilum were similar to a prior study conducted in southwestern Pennsylvania [18]. In spite of these three pathogens potentially sharing some common animal reservoirs, we found B. burgdorferi to have an uneven distribution (independent of sample size) among the nine recreational areas we sampled which could be due to differences in prevalence of hosts these ticks feed on during the larvael or nymphal stages of their development [10]. Additionally, the number and/or source of blood meals, and thus the opportunity for infection, could possibly be the reason for marginal differences in B. burgdorferi $(p=0.054)$ and A. phagocytophilum $(p=0.078)$ prevalence between male and female ticks [10]. The $27 \%$ prevalence rate for $B$. microti in this study could be attributed to regional variation [20] and sampling of $I$. scapularis ticks for microbial pathogens in a high incidence southwestern Pennsylvania county compared to prior studies which sampled ticks from random locations in western Pennsylvania [18,19,21]. In support of our findings, a study by Piesman, et al. [22] demonstrated the wide prevalence of $B$. microti $(2 \%-47 \%)$ in adult $I$. scapularis from four areas in eastern Massachusetts.

Although the biotic and abiotic factors influencing the distribution of I. scapularis ticks and their transmission of the zoonotic pathogens they carry are complex [23] and beyond the scope of this manuscript, it is obvious that Lyme disease in southwest Pennsylvania is on the rise [11,13]. During a five year period (2010-2014), Pennsylvania Department of Health [11] reported a seven fold increase in Lyme disease incidence rate in southwest Pennsylvania (75.72 in 2014 versus 11.5/100,000 persons in 2010) as compared to a two-fold increase in northeast Pennsylvania (54.4 in 2014 versus 30.51 in 2010), which the CDC 


\section{have corroborated in their recent findings [13]}

It has been previously reported that human Lyme and Babesiosis are commonly diagnosed in overlapping geographic areas, especially in regions with high human densities of the northeast (New York, New Jersey) and Midwest (Minnesota, Wisconsin) United States [24]. To date, the human incidence rate for Anaplasmosis and Babesiosis for Pennsylvania is not reported by CDC. But, given our findings and the high incidence rates for Anaplasmosis and Babesiosis in states bordering Pennsylvania (New York and New Jersey) [1], human infection by one of these two emerging blood-borne pathogens is possible via a single I. scapularis tick bite [25] which could result in concurrent infections and contribute to a variety of clinical symptoms not typically seen in Lyme disease patients.

Due to current observed changes in the seasonal and environmental landscape of our planet, the upward trend in $I$. scapularis tick population size and co-infection rates with the zoonotic pathogens is likely to continue [2]. Although factors affecting tick coinfection and risks posed to humans need further study, it is warranted that the scientific community, public health officials, and health care providers, think beyond Lyme so as to be unbiased in their diagnosis of other emerging tick-borne diseases as well as co-infection complications that could result from a single I. scapularis tick bite. We also urge managers and public users of recreational areas to be aware of the risks of coinfections and become more familiar with tick prevention measures and symptoms of these emerging tick-borne diseases.

\section{Acknowledgments}

This study was supported by Indiana University of Pennsylvania Senate Grants to Dr. V. R. Irani (2013-14) and to Dr. V. R. Irani and Dr. Jeffery L. Larkin (2016-17). We thank Dr. Paul M. Nealen for his help with statistical analyses and also thank all involved in collecting and identifying ticks from these Indiana county recreational areas, providing tick and pathogen DNA for positive controls. In addition, we thank Dr. Kugeler for sharing Figure 1 from her 2015 Emerging Infectious Disease publication [13].

\section{References}

1. Center for Disease Control and Prevention (CDC). CDC provides estimate of Americans diagnosed with Lyme disease each year. Available online at http://www.cdc.gov/DataStatistics/

2. Gray JS, Dautel H, Estrada-Peña A, Kahl O, Lindgren E. Effects of climate change on ticks and tick-borne diseases in Europe. Interdiscip Perspect Infect Dis. 2009;2009:593232. doi: 10.1155/2009/593232.

3. De la Fuente J, Estrada-Pena A, Venzal JM, Kocan KM, Sonenshine DE Overview: ticks as vectors of pathogens that cause disease in humans and animals. Front Biosci. 2008;13:6938-46.

4. LoGiudice K, Ostfeld RS, Schmidt KA, Keesing F. The ecology ofinfectious disease: effects of host diversity and community composition on Lyme disease risk. Proc Natl Acad Sci U S A. 2003;100(2):567-71.

5. Hersh MH, Tibbetts M, Strauss M, Ostfeld RS, Keesing F. Reservoir competence of vertebrate hosts for Babesia microti. Emerg Infect Dis. 2012;18(12):1951-7. doi: 10.3201/eid1812.111392.

6. Keesing F, Hersh MH, Tibbetts M, McHenry DJ, Duerr S, Brunner
J, et al. Reservoir competence of vertebrate hosts for Anaplasma phagocytophilum. Emerg Infect Dis. 2012;18(12):2013-6. doi: 10.3201/eid1812.120919.

7. Krause PJ, Telford SR 3rd, Spielman A, Sikand V, Ryan R, Christianson $D$, et al. Concurrent Lyme disease and babesiosis. Evidence for increased severity and duration of illness. JAMA. 1996;275(21):165760.

8. Belongia EA. Epidemiology and impact of coinfections acquired from Ixodes ticks. Vector Borne Zoonotic Dis. 2002;2(4):265-73.

9. Tokarz R, Jain K, Bennett A, Briese T, Lipkin WI. Assessment of polymicrobial infections in ticks in New York State. Vector Borne Zoonotic Dis. 2010;10(3):217-21. doi: 10.1089/vbz.2009.0036.

10. National Academies Press (US) (NAP). Institute of Medicine (US) Committee on Lyme disease and Other Tick-Borne Diseases: The State of the Science. Critical Needs and Gaps in Understanding Prevention, Amelioration, and Resolution of Lyme and Other Tick-Borne Diseases: The Short-Term and Long-Term Outcomes: Workshop Report. Washington (DC). 2011;Available online at http://www.ncbi.nlm.nih. gov/books/NBK57024/

11. Pennsylvania Department of Health (PA DOH). The 2014 Lyme disease Report. Available online at http://www.health.pa.gov/My\%20 Health/Diseases\%20and\%20Conditions/I-L/Pages/Lyme-Disease .aspx\#.Va0Zq7U3nnE. Published June 2015.

12. CourtneyJW, Dryden RL, Montgomery J,Schneider BS, Smith G, Massung RF. Molecular characterization of Anaplasma phagocytophilum and Borrelia burgdorferi in Ixodes scapularis ticks from Pennsylvania. J Clin Microbiol. 2003;41(4):1569-73.

13. Kugeler KJ, Farley GM, Forrester JD, Mead PS. Geographic distribution and expansion of human Lyme disease, United States. Emerg Infect Dis. 2015;21(8):1455-7. doi: 10.3201/eid2108.141878.

14. Pennsylvania Department of Conservation and Natural Resources. The Economic Significance and Impact of Pennsylvania State Parks: An Updated Assessment of 2010 Park Visitor Spending on the State and Local Economy. 2012; Available online http://www.dcnr.state. pa.us/cs/groups/public/documents/document/dcnr_007019.pdf

15. US Fish and Wildlife Service (USFWS) National Fishing and Hunting License Report. 2015. Available online http://wsfrprograms.fws.gov/ Subpages/LicenseInfo/LicenseIndex.htm.

16. Krantz GW, Walter DE. A Manual of Acarology. Third Edition. Oregon State University Book Stores, OR. 2009.

17. Armstrong PM, Katavolos P, Caporale DA, Smith RP, Spielman A, Telford SR 3rd. Diversity of Babesia Infecting Deer Ticks (Ixodes dammini). Am J Trop Med Hyg. 1998;58(6):739-42.

18. Hutchinson ML, Strohecker MD, Simmons TW, Kyle AD, Helwig MW. Prevalence of Borrelia burgdorferi (Spirochaetales: Spirochaetaceae), Anaplasma phagocytophilum (Rickettsiales: Anaplasmataceae), and Babesia microti (Piroplasmida: Babesiidae) in host-seeking Ixodes scapularis (Acari: Ixodidae) from Pennsylvania. J Med Entomol. 2015;52(4):693-8. doi: 10.1093/jme/tjv037.

19. Steiner FE, Pinger RR, Vann CN, Grindle N, Civitello D, Clay K, et al. Infection and co-infection rates of Anaplasma phagocytophilum variants, Babesia spp., Borrelia burgdorferi, and the Rickettsial endosymbiont in Ixodes scapularis (Acari: Ixodidae) from sites in Indiana, Maine, Pennsylvania, and Wisconsin. J Med Entomol. 2008;45(2):289-97.

20.Edwards MJ, Barbalato LA, Makkapati A, Pham KD, Bugbee LM. Relatively low prevalence of Babesia microti and Anaplasma 
phagocytophilum in Ixodes scapularis ticks collected in the Lehigh valley region of eastern Pennsylvania. Ticks Tick Borne Dis. 2015;6(6):812-9. doi: 10.1016/j.ttbdis.2015.07.009.

21. Brown SM, Lehman PM, Kern RA, Henning JD. Detection of Borrelia burgdorferi and Anaplasma phagocytophilum in the black-legged tick, Ixodes scapularis, within southwestern Pennsylvania. J Vector Ecol. 2015;40(1):180-3. doi: 10.1111/jvec.12148.

22. Piesman J, Mather TN, Donahue JG, Levine J, Campbell JD, Karakashian SJ, et al. Comparative prevalence of Babesia microti and Borrelia burgdorferi in four populations of Ixodes dammini in eastern Massachusetts. Acta Trop. 1986;43(3):263-70.
23. Shaw MT, Keesing F, McGrail R, Ostfeld RS. Factors influencing the distribution of larval blacklegged ticks on rodent hosts. Am J Trop Med Hyg. 2003;68(4):447-52.

24. Knapp KL, Rice NA. Human coinfection with Borrelia burgdorferi and Babesia microti in the United States. JParasitol Res. 2015;2015:587131. doi: $10.1155 / 2015 / 587131$.

25. Piesman J, Hicks TC, Sinsky RJ, Obiri G. Simultaneous transmission of Borrelia burgdorferi and Babesia microti by individual nymphal Ixodes dammini ticks. J Clin Microbiol. 1987;25(10):2012-13. 